\title{
Special issue on whole-body control of contacts and dynamics for humanoid robots
}

\author{
Serena Ivaldi $^{1}$ - Jan Babič ${ }^{2} \cdot$ Michael Mistry $^{3} \cdot$ Robin Murphy ${ }^{4}$
}

Received: 25 December 2015 / Accepted: 9 January 2016 / Published online: 27 January 2016

(C) Springer Science+Business Media New York 2016

Whether you are walking on a concrete floor, standing on a carpet, or sitting on a soft chair, your entire body continuously controls the posture and the contact forces that are produced by acting on rigid and compliant surfaces. Sometimes, like when reaching for a distant object or standing inside a moving bus that suddenly brakes, humans plan intentionally new contacts to preserve their balance and avoid falling.

For humanoid robots to act in unstructured natural environments as humans do, contacts and physical interactions are necessary and unavoidable. In recent years, whole-body control techniques have matured to the point where various humanoid robots can robustly interact with their environment. Robots may exploit predictable contacts to aid in goal achievement, as well as learn dynamics of contact to generalize over novel tasks and domains. They may regulate their compliance to cope with unpredictable contacts and ensure safe behaviors. While these achievements are a major milestone for robotics, they still need to be applied to more challenging situations, inspired by natural settings and physical interaction scenarios. There is a strong need for advanced methods that can handle multiple contacts, unforeseen or

Serena Ivaldi

serena.ivaldi@inria.fr

Jan Babič

jan.babic@ijs.si

Michael Mistry

m.n.mistry@bham.ac.uk

Robin Murphy

murphy@cse.tamu.edu

Inria, 54600 Villers-lès-Nancy, France

2 Jožef Stefan Institute, Ljubljana, Slovenia

3 University of Birmingham, Birmingham, UK

4 Texas A\&M University, College Station, USA intentional, with different rigidity properties, and guarantee the robust, autonomous execution of actions (balancing, walking, manipulation) in variable contexts.

Such a need has never been as evident as was in the DARPA Robotics Challenge (DRC), an international competition funded by the US Defense Advanced Research Projects Agency. During the DRC, several international teams were remotely controlling a semi-autonomous humanoid robot to perform whole-body tasks that involved contacts, such as walking, driving a car and climbing stairs. The DRC spurred the development of new humanoid robots and advances in both mechanical design, hardware and software development. Independently of the DRC, many projects addressing control of contacts were funded by the European Commission, such as CODYCO, ${ }^{1}$ COGIMON $^{2}$ and WALKMAN. ${ }^{3}$ The latter was also instrumental to the design and building of the homonym humanoid robot that participated to the DRC.

This increased interest for whole-body control has also become apparent through the proliferation of several international workshops at the most important robotics conferences, such as the well attended workshop "Whole-body Compliant Dynamical Contacts for Humanoid Robotics" at ICRA 2013 in Karlsruhe, Germany. ${ }^{4}$ Several workshops followed up, such as the "Torque-Controlled Humanoids" in HUMANOIDS $2013^{5}$ and the workshop "Towards a Unifying Framework for Whole-body and Manipulation Control" in RSS $2015 .{ }^{6}$

\footnotetext{
${ }^{1} \mathrm{http} / / / \mathrm{www}$. codyco.eu/.

$2 \mathrm{http} / / /$ cogimon.eu/.

${ }^{3}$ http://www.walk-man.eu/.

${ }^{4}$ http://www.codyco.eu/index.php/workshop-icra2013.

5 http://www.codyco.eu/index.php/workshop-humanoids2013.

${ }^{6}$ http://www.codyco.eu/index.php/45-workshop-rss2015.
} 

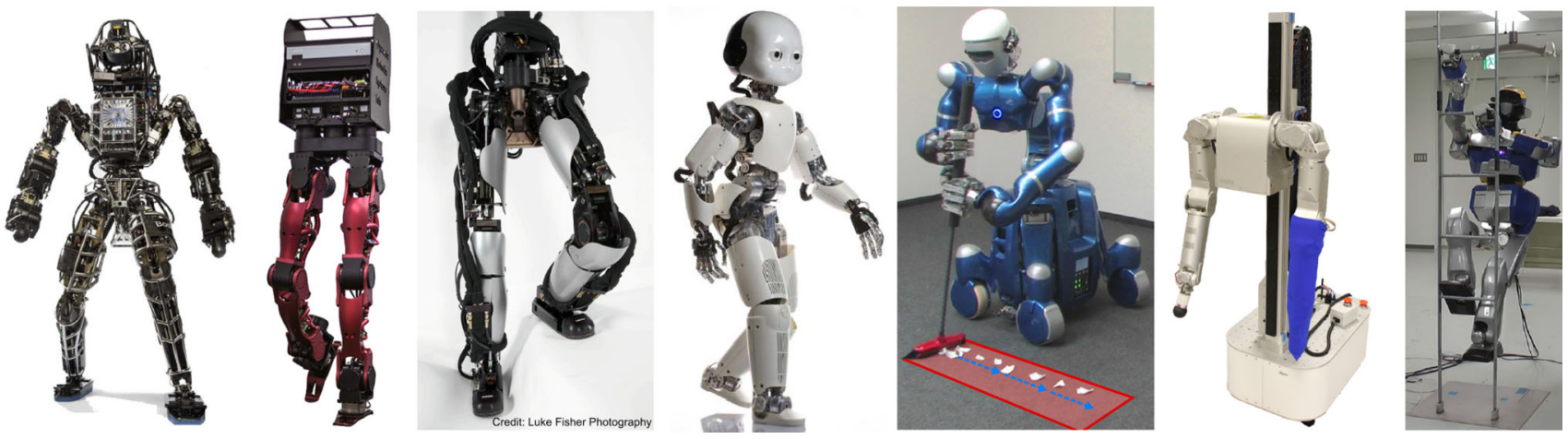

Fig. 1 The robots that were used in the selected papers of this special issue. From left to right Atlas, Dyros, Sarcos, iCub, Justin, Darci and HRP2

This special issue of the Autonomous Robots journal aims at presenting the advances in whole-body control of real robots, focusing on control and learning techniques applied to estimation, control and adaptation of whole-body dynamics movement and contact forces that go beyond basic balancing abilities. The special issue received the support of the IEEERAS Technical Committee in Whole-Body Control through advertisement on its website. ${ }^{7}$ Papers were solicited with an open call that was advertised about six months before the deadline. A deadline extension was then allowed, as many authors were still engaged in the DRC. We received more than thirty submissions to the special issue, which were rigorously reviewed by up to four reviewers, as well as by at least one of the guest editors. Eight papers were selected for this special issue. We consciously decided to narrow the impressive pool of submissions to those papers which presented relevant research and success stories with real robotics platforms, shown in Fig. 1.

The paper "Optimization-based locomotion planning, estimation and control design for the Atlas humanoid robot" by Kuindersma et al., presents an overview of the optimization algorithms for planning and control that were developed for their Atlas robot competing in the DRC. Particularly, they describe a sparse nonlinear trajectory optimization algorithm that combines full body kinematics with centroidal dynamics to efficiently compute whole-body motions in presence of multiple contacts. Their controller relies on reduced dynamical models to construct an efficiently-solvable quadratic programming problem with active-sets, which is able to achieve stable walking and whole-body trajectories despite the inevitable model inaccuracies and sensor noise. The input to the controller is a state estimator that fuses information from the several sensors of the Atlas robots, namely kinematic, inertial and LIDAR information. The paper reports on interesting walking experiments performed with the Atlas robot, while it discusses simulations about very dynamics motions such as running and jumping.

\footnotetext{
${ }^{7} \mathrm{http} / / /$ wholebodycontrol.eu/.
}

The paper "Balancing of humanoid robot using contact force/moment control by task-oriented whole-body control framework" by Lee et al., presents a balance controller for the torque-controlled legged robot Dyros. The proposed controller combines the desired joint torques computed by the multi-task whole-body control framework of Park \& Khatib with the control of contact forces and moments, exploiting the null-space of the high-priority task. Interestingly, their approach allows the robot to redistribute the contact forces and moments, thus enabling a better contact transition from single to double support phase. Several balancing experiments with the robot Dyros are discussed.

Herzog et al. present a "Momentum control with hierarchical inverse dynamics on a torque-controlled humanoid". Their work focuses on practical implementation of a cascade of QPs for finding an inverse dynamics solution for multiple task constraints. Real-time performance is achieved, and furthermore, feedback gains are optimised with an LQR design. Their momentum based balance controller is rigorously tested on the lower body of the torque-controlled Sarcos Humanoid Robot, in both single and double support, and comparisons are made with other balancing approaches.

Another multi-task hierarchical controller, applied on the iCub humanoid robot, is presented by Liu et al. in the paper titled "Whole-body hierarchical motion and force control for humanoid robots". Their approach uses a generalised projector with time varying weights based on the augmented Jacobian. Weights can be adjusted to specify the (strict or non-strict) priority between tasks, and the scheme finds an optimal solution solving only a single QP. Experiments for their torque-based quasi-static control framework are shown using both a simulated and real iCub humanoid.

Two papers describe whole-body control strategies for the Justin robot. The first by Dietrich et al. titled "Whole-body impedance control of wheeled mobile manipulators: stability analysis and experiments on the humanoid robot Rollin' Justin" extends whole-body impedance control approaches to systems with mobile bases. In their method, the torquecontrolled upper body is decoupled from the admittance- 
controlled wheeled base by compensating for the inertia and Coriolis/centrifugal couplings between the two subsystems. The result is a symmetrical mass matrix and a provably stable passive control loop. Experiments show a human physically interacting with the Rollin' Justin platform.

The second by Leidner et al. titled "Knowledge-enabled parameterization of whole-body control strategies for compliant service robots" proposes a reasoning framework for the execution of force-sensitive manipulation tasks using a humanoid robot. The main contribution of the paper is the utilization of low level compliant whole-body control strategies parameterized by high level hybrid reasoning mechanisms. The paper provides a classification of different wiping tasks, deals with low-level control mechanisms to carry out the necessary movements, and describes the associated action templates, which need to be parametrized to perform the task in the given context.

The paper "Model predictive control for fast reaching in clutter" by Killpack et al. presents a model predictive control scheme for arm movements in cluttered environments. The tactile sensors that completely cover the robot's arm surface are used for detection and approximation of contacts. This contact information is then taken into account inside the problem formulation, which tries to reach the goal while avoiding large contact forces. The main contribution of the paper is the inclusion of the full dynamic model of the robot and the extension of the control horizon to multiple time steps which allows for faster reaching. Several simulations and real robot experiments are used to demonstrate performance improvements over the previous quasi-static model predictive control schemes.

Finally, the paper "Multi-contact vertical ladders climbing with a HRP-2 humanoid" by Vaillant et al. describes the methodological and experimental approaches undertaken to enable a full size humanoid robot to climb on a vertical industrial ladder. The described methodological approach combines several methods for planning and control. A multi-contact planner is applied for posture generation where gripper torque optimization is utilized for computing different static postures during climbing the ladder. Multiobjective quadratic-program is used to map the resulting plan of contact transitions and postures to joint trajectories that serve as set points for the low-level joint controller of the HRP-2 robot.

All the eight papers present significant advances in the state of the art in whole-body control from both theoretical and experimental point of view. The results that the authors achieved with their respective robots are commendable. We would like to thank all the reviewers who provided excellent review reports of the papers, and helped us in selecting the best papers among the many very interesting papers that were submitted. We hope that the readers will enjoy our selection for this special issue on whole-body control of contacts and dynamics for humanoid robots.

Acknowledgments This work was supported by the European Community through the FP7 EU Project CoDyCo (No. 600716 ICT 2011.2.1 Cognitive Systems and Robotics).

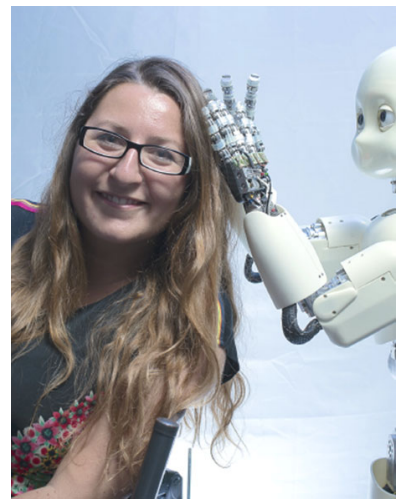

Serena Ivaldi is a researcher in INRIA, France. She received the B.S. and M.S. degree in Computer Engineering, both with highest honors, at the University of Genoa (Italy) and her Ph.D. in Humanoid Technologies in 2011, jointly at the University of Genoa and the Italian Institute of Technology. There she also held a research fellowship in the Robotics, Brain and Cognitive Sciences Department. She was a postdoctoral researcher in the Institut des Systèmes Intelligents et de

Robotique (ISIR) in University Pierre et Marie Curie, Paris, then in the Intelligent Autonomous Systems Laboratory in the Technical University of Darmstadt, Germany. Since November 2014, she is a researcher in INRIA Nancy Grand-Est, in the robotics team Larsen. Her research is centered on robots interacting physically and socially with humans, blending learning, perception and control.

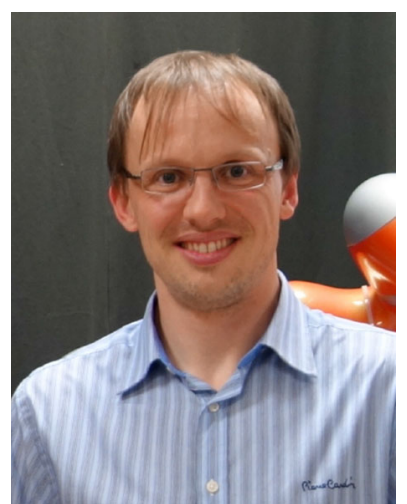

Jan Babič is a Senior Researcher at Jožef Stefan Institute in Slovenia. He received his $\mathrm{Ph} . \mathrm{D}$. from Faculty of Electrical Engineering, University in Ljubljana examining the role of biarticular muscles in human locomotion. During the years 2006/2007 he was a visiting researcher at ATR Computational Neuroscience Laboratories in Japan. In November 2014 he was a visiting professor at The Institute for Intelligent Systems and Robotics, University of

Pierre and Marie Curie in France. His research is concerned with the understanding how human brain controls movement of the body. A main focus of his research is to understand how the central nervous system process sensory information and transfer them to motor commands. He is especially interested in developing models of full-body motor learning that help to elucidate the underlying neural mechanisms by which the brain iteratively modifies muscle activations during learning so that appropriate forces and viscoelastic impedance are created to ensure the stability. These models are then used to design biologically plausible solutions for a broad spectrum of robotic systems such as industrial robots, humanoids, exoskeletons and rehabilitation devices. 


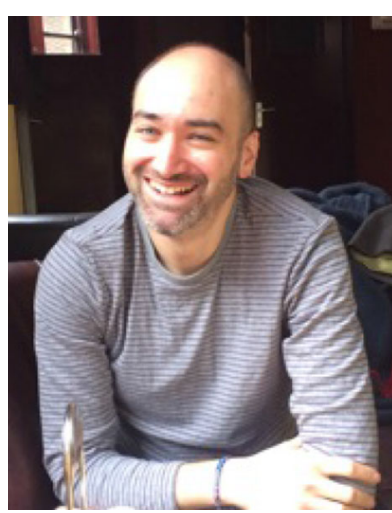

Michael Mistry is a Senior Lecturer in Robotics at the School of Computer Science, University of Birmingham, where he is also a member of the Intelligent Robotics Lab and the Centre for Computational Neuroscience and Cognitive Robotics. He is broadly interested in human motion and humanoid robotics. His research focuses on issues relevant to dexterous movement in both humans and humanoid robots, including redundancy resolution and inverse kinematics, operational space control and manipulation, stochastic optimal control, and internal model learning and control, particularly in environmental contact. Previously, he has been a postdoc at the Disney Research Lab at Carnegie Mellon University, a researcher at the ATR Computational Neuroscience Lab, and a Phd student in Stefan Schaal's CLMC lab at the University of Southern California.

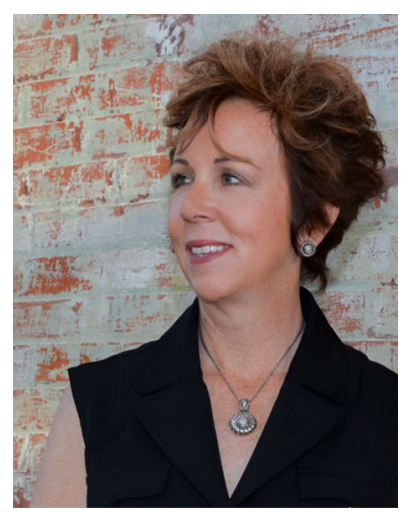

Robin Murphy is the Director of Center for Robot-Assisted Search and Rescue (CRASAR), the Founder of Roboticists Without Borders and a Raytheon Professor of Computer Science and Engineering. She is Engineering Faculty Fellow for Innovation in High-Impact Learning Experiences, and Recipient of 2014 Eugene L. Lawler Award for Humanitarian Contributions within Computer Science and Informatics. She is a IEEE Fellow, a Drone Ranger, Fast Company's Most Influential Women of 2011, Alpha Geek in WIRED (17.07), Top 25 Doers, Dreamers and Drivers for 2015 by Government Technology Magazine. 